\title{
THE GENERALIZED POINCARE CONJECTURE IN HIGHER DIMENSIONS
}

\author{
BY STEPHEN SMALE ${ }^{1}$
}

Communicated by Edwin Moise, May 20, 1960

The Poincaré conjecture says that every simply connected closed 3 -manifold is homeomorphic to the 3 -sphere $S^{3}$. This has never been proved or disproved. The problem of showing whether every closed simply connected $n$-manifold which has the homology groups of $S^{n}$, or equivalently is a homotopy sphere, is homeomorphic to $S^{n}$, has been called the generalized Poincaré conjecture.

We prove the following theorem.

THEOREM A. If $M^{n}$ is a closed differentiable $\left(C^{\infty}\right)$ manifold which is a homotopy sphere, and if $n \neq 3,4$, then $M^{n}$ is homeomorphic to $S^{n}$.

We would expect that our methods will yield Theorem A for combinatorial manifolds as well, but this has not been done.

The complete proof will be given elsewhere. Here we give an outline of the proof and mention other related and more general results.

The first step in the proof is the construction of a nice cellular type structure on any closed $C^{\infty}$ manifold $M$. More precisely, define a real valued $f$ on $M$ to be a nice function if it possesses only nondegenerate critical points and for each critical point $\beta, f(\beta)=\lambda(\beta)$, the index of $\beta$.

TheOREM B. On every closed $C^{\infty}$ manifold there exist nice functions.

The proof of Theorem B is begun in our article [3]. In the terminology of [3], it is proved that a gradient system can be $C^{1}$ approximated by a system with stable and unstable manifolds having normal intersection with each other. This is the announced Theorem 1.2 of [3]. From this approximation we are then able to construct the function of Theorem B.

The stable manifolds of the critical points of a nice function can be thought of as cells of a complex while the unstable manifolds are the duals. This structure has the advantage over previous structures that both the cells and the duals are differentiably imbedded in $M$. We believe in fact that nice functions will replace much of the use of $C^{1}$ triangulations and combinatorial methods in differential topology.

\footnotetext{
${ }^{1}$ Supported by a National Science Foundation Postdoctoral fellowship.
} 
In our work here we do not actually use the cells themselves as much as the neighborhoods of the skeletons of this structure. More precisely, if $f$ is a nice function on $M$, let $X_{k}=f^{-1}[0, k+1 / 2]$.

Then each $X_{k}$ is a compact $C^{\infty}$ manifold with boundary and one can think of $X_{k}$ as being obtained from $X_{k-1}$ by attaching a number of $k$-cells and "thickening" them.

If $M$ is a manifold with boundary, $D$ a disk, $f: \partial D \rightarrow \partial M$ a differentiable imbedding with a trivial normal bundle $B$ and $\gamma$ a homotopy class of cross-sections $\partial D \rightarrow B$, then a new manifold $M \cup D=M \cup_{f, \gamma} D$ is defined. We do not define $M \cup D$ here but only remark that $M \cup D$ is roughly $M$ with $D$ attached by $f$, thickened according to $\gamma$, and smoothed.

Then it can be shown that $X_{k}=X_{k-1} \cup D_{1}^{k} \cup \ldots \cup D_{r}^{k}$, where $\operatorname{dim} D_{i}^{k}=k\left(D, D^{k}\right.$, etc. always denote disks).

The handlebodies are defined to be all manifolds of the form $D^{n} \cup D_{1}^{s} \cup \ldots \cup D_{k}^{s}$. Fixing $n, k, s$, the set of all such manifolds is denoted by $\mathfrak{H C}(n, k, s)$.

For our main theorems we prove that under homotopy assumptions, the $X_{k}$ are handlebodies. The following is a major step.

TheOREM C (THE HANDLEBOdy THEOREM). Let $n \geqq \max (2 s+2,5)$, $H \in \mathfrak{F}(n, k, s), K=H \cup D_{1} \cup \ldots \cup D_{r}, \operatorname{dim} D_{i}=s+1$ and $\pi_{s}(K)=0$. Also assume $\pi_{1}\left(H \cup D_{1} \cup \cdots \cup D_{r-k}\right)=1$ if $s=1$. Then $K \in \mathfrak{F}(n, r-k, s+1)$.

We shall not try to summarize the proof of Theorem C.

Using Theorems $\mathrm{B}$ and $\mathrm{C}$ and the previous consideration one can obtain the following theorem.

Theorem D. Let $M^{n}$ be a closed $C^{\infty}$ manifold which is $(m-1)$ connected and $n \geqq \max (2 m, 5)$. Then there is a nice function $f$ on $M$ with type numbers satisfying

$M_{0}=M_{n}=1$ and $M_{i}=0$ for $0<i<m$ and $n-m<i<n$.

Special cases of Theorem $\mathrm{D}$ are first Theorem $\mathrm{E}$ if $n=2 m+1$, and Theorem $\mathrm{F}$ if $n=2 m$.

Theorem E. Let $M$ be a closed ( $m-1)$-connected closed $C^{\infty}(2 m+1)$ manifold. Then $M=H \cup H^{\prime}$ where $H, H^{\prime} \in \mathfrak{K C}(2 m+1, k, m)$.

This generalizes the Heegard decomposition of a 3-manifold.

Theorem F. Let $M$ be a closed $(m-1)$-connected $C^{\infty} 2 m$-manifold, $m \neq 2$. Then there is a nice function on $M$ whose type numbers equal the Betti numbers of $M$. 
Theorem $\mathrm{F}$ implies the even dimensional part of Theorem A.

According to Thom (see [2]) two closed oriented $C^{\infty}$ manifolds $M_{1}$ and $M_{2}$ are $J$-equivalent if there exists a $C^{\infty}$ manifold $X$ with boundary $M_{1}-M_{2}$ and each $M_{i}$ is a deformation retract of $X$. Using methods similar to the preceding ones we are able to prove the following:

Theorem G. If two homotopy spheres $M_{1}$ and $M_{2}$ of dimension $2 m-1, m \neq 2$ are $J$-equivalent, then they are diffeomorphic.

Using Mazur's Theorem [1], the part of Theorem A for odd dimensional manifolds follows from Theorem $\mathrm{G}$.

Theorem H. There exists a triangulated manifold with no differentiable structure at all.

This follows from Theorem $\mathrm{G}$ and work of Milnor [2]. Take his manifold of $W_{0}$ of Theorem 4.1 of [2] for $k=3$ and attach a $4 k$ cell. (In our context $W_{0}$ can be viewed as a certain handlebody in $\mathfrak{H}(4 k, 8,2 k)$.) The following also follows from Milnor [2] and Theorem $\mathrm{G}$ :

THEOREM I. The groups $\Gamma^{2 m+1}$ are finite, for all $m$.

For example, for $\Gamma^{5}=0$, there are precisely 28 differentiable structures on $S^{7}$, etc.

\section{REFERENCES}

1. B. Mazur, On embeddings of spheres, Bull. Amer. Math. Soc. vol. 65 (1959) pp. 59-65.

2. J. Milnor, Differentiable manifolds which are homotopy spheres, (mimeographed) Princeton University, 1959.

3. S. Smale, Morse inequalities for a dynamical system, Bull. Amer. Math. Soc. vol. 66 (1960) pp. 43-49.

Institute for Advanced Study and

Instituto de Matemática, Rio de Janeiro, BraziL 